\title{
Sonia ou le sens retrouvé. Notes sur la rédemption et le salut dans Crime et châtiment
}

\author{
Sonia and the rediscovered sense. Notes on \\ redemption and salvation in Crime and Punishment
}

Samuel Bidaud ${ }^{1}$

\section{Resumen:}

Este artículo propone un nuevo acercamiento a los temas de la redención y de la salvación en Crimen y castigo a través del análisis del personaje de Sonia. Destacaremos que la novela de Dostoïevski está estructurada por una serie de interrogaciones sobre el bien y la salvación terrestre y divina a los cuales Sonia da nuevas respuestas y nuevo sentido. Estas interrogaciones son las siguientes: ¿en qué medida los hombres deben ser buenos? ¿cuáles son las condiciones de la salvación terrestre? Y, finalmente, ¿hay una salvación divina universal para todos los hombres, incluso los peores?

Palabras claves:

Dostoïevski; Crimen y castigo; redención; salvación; SoniaAbstract:

Abstract:

This article proposes a new approach to the themes of redemption and salvation in Crime and punishment by analyzing the character of Sonia. We will notably highlight that the novel of Dostoïevski is structured with a series of questions about good and earthly and divine salvation to which Sonia gives new perspectives and meanings. These are the following questions: To what extent should men be good? What are the conditions of salvation on earth? And, finally, is there a universal and divine salvation for all men, even the worst?

\section{Keywords :}

Dostoïevski; Crime and punishment, redemption; salvation; Sonia.

Artículo recebido el 27 de mayo de 2012 y aprobado el 8 de febrero de 2013

1 Université de Bourgogne , France. Correo electrónico: samuel.bidaud@aliceadsl.fr 
On a souvent privilégié le personnage de Raskolnikov dans les études sur Crime et châtiment, notamment lorsque l'on insistait sur l'aspect policier du roman de Dostoïevski². Or, la lecture psychologique et religieuse de Crime et châtiment que l'on voudrait faire ici (la lecture humaniste, osons le mot), et la recherche de sa valeur (au sens de Vincent Jouve ${ }^{3}$ ), impliquent également de s'intéresser à Sonia, la sainte qui amène Raskolnikov à revivre dans l'amour. Certes, la thématique de la rédemption dans Crime et châtiment a été déjà très largement étudiée. Toutefois, c'est dans une perspective plus large que nous voudrions ici l'intégrer. En effet, Crime et châtiment apporte non seulement une réponse à la question du sens de la souffrance consentie et rédemptrice, mais également à d'autres questions qui, si elles sont a priori moins visibles dans le roman de Dostoïevski, n’en sont pas moins présentes. Ce sont ces questions qui tourmentent lécrivain que nous voudrions ici mettre au jour et étudier rapidement. Or, ces interrogations qui sont liées les unes aux autres concernent aussi bien l'idéal de sainteté que représente Sonia que les conditions de la souffrance et la nature de la rédemption et du salut. Posons-les de la manière suivante : jusqu’à quel point l'homme, ou plutôt ici la femme, Sonia, doit-elle être bonne? Y a-t-il un salut humain possible pour tous les hommes? Et y a-t-il un salut divin certain et universel pour l'ensemble des hommes, même les pires? Toutes ces questions trouvent une réponse notamment dans le personnage de Sonia, qui illumine le roman et donne à la vie un sens neuf deux ans après les Carnets du sous-sol4.

L'importance de Sonia et de la figure de sainte qu'elle représente dans Crime et châtiment convient d'être d’emblée analysée. On n’a pas suffisamment

2 Voir par exemple De Grève (1998).

3 II s'agira en effet, dans la mesure où «tout texte suppose un point de vue" (Jouve, 2001, p. 9), d'analyser la «mise en texte des valeurs».

4 Observons que les Carnets $d u$ sous-sol mettent également en scène un homme qui se décrit comme malade et méchant, et une prostituée qui essaye à la fin du récit de le sauver sans y parvenir. Toutefois le narrateur n'est pas un criminel, comme Raskolnikov, et la jeune prostituée, Liza, n'est pas comme Sonia, et finit par fuir, effrayée, devant le mal du narrateur. Les caractères ne sont donc pas poussés à l'extrême comme dans Crime et châtiment. souligné en effet le caractère extrême de cette sainteté, qui dépasse de loin la bonté et la tendresse déjà présentes chez d’autres personnages féminins avant Crime et châtiment, qu'il s'agisse de Mme M... dans Un petit héros ${ }^{5}$ ou d'Alexandra Mikhaïlovna dans Netotchka Nezvanova ${ }^{6}$, et qui ne peut trouver d'équivalent que dans le prince Muyshkine.

Sonia s'est sacrifiée pour sa famille en se prostituant, elle se sacrifie pour les autres en permanence, et elle ne veut jamais de mal à personne; elle rend le bien pour le mal, et serait prête à donner sa vie pour n'importe quel homme, même le pire. Toute sa vie n'est que sacrifice et amour désintéressé pour l'ensemble des hommes, dans l'humilité la plus complète.

Une relecture de tous les passages du roman où Sonia apparaît nous montre que la jeune fille n'a jamais un mot dur pour quiconque et qu'elle est pleine de pardon pour ceux qui peuvent lui faire du mal. Ainsi lorsque Raskolnikov se rend chez elle juste après la mort de Marmeladov et qu'il en vient à évoquer les coups que Catherine Ivanovna a pu donner à Sonia, cette dernière répond à Raskolnikov qu'il se trompe et est pleine de compassion pour sa malheureuse belle-mère dont elle fait léloge: «Il était

5 Voici par exemple la description de Mme M... au début de Un petit héros: " Mais la belle jeune femme paraissait taciturne, renfermée, bien qu'il n'y eût certes personne de plus attentif et de plus aimant envers quiconque avait besoin de compassion. Il y a des femmes qui sont dans la vie comme des soeurs de charité. [...] Celui qui souffre peut hardiment et avec espoir aller à elles, sans crainte d'être importun, car peu d'entre nous savent ce qu'il peut y avoir d'amour infiniment patient, de commisération et d'indulgence sans bornes dans certains coeurs féminins. " (Dostoïevski, 2010, p. 49).

6 Ainsi Netotchka écrit-elle par exemple à propos d'Alexandra Mikhaïlovna que I'on ressentait " l'amour qu'irradiait son regard tendre, ses deux grands yeux bleu clair, son sourire timide et tout ce visage doux et pâle qui reflétait parfois tant de candeur, tant de naïveté, quelque chose de tellement sans défense, qui semblait craindre comme pour la moindre de ses sensations, le moindre élan de son coeur - la joie d'un seul instant ou la tristesse timide et coutumière. Mais, parfois, pendant une minute heureuse, sans inquiétude, on voyait dans ce regard qui vous prenait au coeur tant de clarté, tant de lumière, comme celle du plein jour, tant de sérénité sans tache; ces yeux, bleus comme le ciel, brillaient d'un tel amour, avaient un regard si doux, ils reflétaient toujours un sentiment de sympathie si profond envers tout ce qui était noble, tout ce qui demandait de l'amour, priait de la compassion, - que toute l'âme se soumettait à elle, s'élançait vers elle comme malgré soi et, semblait-il, reprenait d'elle cette clarté, cette tranquillité de l'âme, l'accord avec le monde, et l'amour. " (Dostoïevski, 2000, p. 207). 
visible [...] qu'elle avait terriblement envie [...] de prendre la défense de sa marâtre. Une compassion insatiable [...] se refléta soudain sur tous les traits de son visage» (Dostoïevski, 1984, p. 369).

Ce principe évangélique de tendre l’autre joue et de donner le bien pour le mal culmine lorsque Raskolnikov avoue à Sonia le meurtre de l'usurière et de sa soeur Lizaveta, l'amie de Sonia. La réaction de Sonia est en effet de se jeter dans les bras de Raskolnikov : "Qu'avez-vous fait... qu'avez-vous fait là de vous-même! prononça-t-elle avec désespoir, et se redressant, elle se jeta à son cou, le prit et le serra fortement, fortement dans ses bras», et la jeune fille est emplie d'un sentiment de compassion immense pour celui qui a assassiné son amie : «Non, il n'y a pas plus malheureux que toi, à cette heure, sur toute la terre!». Et lorsque Raskolnikov qui s'est mis à pleurer demande : «Alors, tu ne m'abandonneras pas, Sonia?», elle répond: «Non, non; jamais, ni nulle part! [...] Je te suivrai, je te suivrai partout! O Seigneur![...] Et pourquoi ne t'ai-je pas connu plus tôt», et elle conclut : «Ensemble, ensemble!... [...] Avec toi j'irai au bagne! Ensemble!» (Dostoïevski, 1984, p. 475).

Ensemble : la réaction de Sonia devant le mal et le crime n'est pas de séloigner du criminel, mais au contraire de s'unir à lui et de lui donner toute sa compassion, dêtre compatissante avec les hommes qui ont fait le mal, et de les aider à revenir vers le bien. Il s'agit aussi bien de la parabole de la brebis égarée que de tendre l'autre joue aux personnes qui font du mal et de faire du bien aux méchants7.

Car Sonia est véritablement une sainte qui se préoccupe de l'ensemble des hommes et qui ne veut de mal à personne, pas même aux pires des

7 Jacques Catteau, dans le tome 2 de la Correspondance de Dostoïevski, précise, à propos d'une lettre de ce dernier à Nikolaï Alexeievitch Lioubimov où l'écrivain demande aux éditeurs de ne pas l'obliger à de nouveaux remaniements de Crime et châtiment, que " Dostoïevski a revu le chapitre 9 [...], contesté par Kratkov en raison surtout de l'excessive idéalisation du personnage de Sonia, "poussant l'abnégation jusqu'à se prostituer pour sa famille" et s'arrogeant le droit de lire l'Evangile à Raskolnikov qui, de son côté, défendrait trop âprement sa conduite criminelle. [...] Dans la version initiale, le dialogue entre Raskolnikov et Sonia était certainement plus fouillé et plus développé " (Catteau, 2000, p. 156). hommes. La jeune fille est bonne à l'extrême, et cette bonté est sondée par Raskolnikov lorsqu'il imagine une situation imaginaire où Sonia aurait le choix entre sauver Catherine Ivanovna et ses enfants et Pierre Loujine qui vient de l'offenser, et où si Sonia laissait vivre Loujine alors Catherine Ivanovna et les orphelins mourraient. Et Raskolnikov l'interroge : qui sauverait-elle? Cette question semble avoir profondément tourmenté Dostoïevski, et la réponse que lui donne Sonia est la seule réponse qu'une sainte pourrait donner : elle ne tuerait personne, et il est vain de demander des choses qui ne peuvent pas arriver, puisque la vie humaine n'appartient quà Dieu : «Pourquoi me demandez-vous ce qui ne peut pas être?», "A quoi bon demander ce qu'il n'est pas permis de demander? Pourquoi des questions aussi vaines?» (Dostoïevski, 1984, p. 469). En d'autres termes, et pour reprendre le problème d'une manière positiviste comme le ferait Bertrand Russell (dont le logicisme ne coïncide que sur ce point avec Dostoïevski bien sûr !) : il n'y a pas de $\mathrm{x}$ tel que $\mathrm{x}$ soit la situation décrite par Raskolnikov. Frédérique Leichter-Flack (2008, p. 76) voit dans l'interrogation de Raskolnikov un moyen pour ce dernier de justifier son crime et propose une analyse rhétorique :

Mais ce dilemme vise à faire endosser à Sonia la justification de son propre crime. Soit elle se décide, et elle légitime le principe " une mort contre cent vies » : Raskolnikov, venu pour avouer son crime, peut alors recevoir l'absolution pour son acte courageux et légitime. Soit elle décide de ne rien décider, de ne pas agir, et alors elle consent à la mort de Katérina et des enfants. Contre ce dilemme destiné à l'enfermer dans un piège, Sonia se défend sur le plan des prémisses qu'elle conteste $[\ldots]$

Mais c'est la conclusion qu'en tire Frédérique Leichter-Flack à laquelle nous ne saurions souscrire : ce nest pas la mauvaise foi de Raskolnikov qui se trouve dévoilée, mais l'une des questions qui semblent avoir tourmenté Dostoïevski.

Sonia est prête à donner sa vie pour n'importe qui, et elle refuse de faire du mal à qui que ce soit dans quelque circonstance que ce soit; et si l' interrogation imaginaire que nous venons d'évoquer 
tourmente Dostoïevski, Sonia lui donne la seule réponse admissible qui la préserve pure de tout mal : il n'y a pas de situation telle qui puisse se produire, où ce serait à elle de décider de la vie de quelqu'un; elle s'y refuse, et refuse d'avoir à choisir, même fictivement, la mort d'un être. Sonia est ainsi sainte jusqu'au bout : non seulement elle est prête à donner sa vie pour n'importe qui, mais en outre elle refuse de faire du mal même au pire des hommes qui existerait, et reconnaît le droit à la vie pour tous. Elle représente là l'exact contraire de Raskolnikov et de la théorie avancée par ce dernier sur Napoléon et le droit de tuer quelqu'un pour faire soi-disant avancer l'humanité; il n'est pas permis de faire couler le sang, quel qu'il soit, d'où le refus systématique par Dostoïevski de tout nihilisme et le rappel que la vie humaine est sacrée. Nous reprenons donc les propos de Mochulski que cite à propos des Pauvres gens Isabel Martínez Fernández (2003, p. 64): «En ese "todos somos hermanos", se ubica el ideal fraternal dostoievskiano, donde siempre tienen cabida tanto los buenos como los malos, ya que, para él, en la comunidad, incluso "el último y el más marginado, también es un hombre, y se llama, mi hermano".»

Dostoïevski a poussé la sainteté jusqu’au bout dans Sonia, jusqu’à apporter des réponses aux situations imaginaires qui le tourmentaient lui-même, parmi lesquelles celle de savoir s'il fallait tuer le pire des criminels si l’on était persuadé que ce dernier tuerait quelqu'un à son tour. Or, c'est là raisonner sur des situations qui nexistent pas, littéralement : jamais nous ne sommes placés dans une situation telle, et il faut refuser d'y répondre; mais, au-delà, le principe fondamental est toujours qu'il ne faut faire de mal à personne. On notera d'ailleurs que si Sonia conseille à Raskolnikov d'aller se dénoncer, c'est parce que ce qu'il risque c'est le bagne, et non la peine de mort, châtiment inacceptable (qu'on songe aux discours du prince Muichkine dans L'Idiot sur le sujet8). Mais nous y reviendrons.

8 Voir L'Idiot (Dostoïevski, 1972, tome 1, p. 33 et suivantes, et p. 95 et suivantes).
La sainteté de Sonia est tout évangélique et marquée par la loi du pardon et de la rédemption qui est annoncée par le Christ dans le Sermon sur la montagne. Rendez le bien pour le mal, ce ne sera pas oeil pour oeil et dent pour dent : voilà la justice qui anime Sonia. Déjà dans les Carnets de la maison morte, le personnage d'Aleï déclarait au narrateur, après que celui-ci lui avait demandé ce qui lui plaisait le plus dans le Sermon : «Là où il (Jésus) dit : pardonne, aime, n'offense pas, et aime tes ennemis. Ah, comme c'est bien, ce qu'il dit !» (Dostoïevski, 1999, p. 122). Or, il nous faut à présent nous interroger sur le sentiment religieux de Sonia au sujet de la rédemption, laquelle est au coeur de Crime et châtiment.

C'est Sonia qui amène Raskolnikov à confesser son crime, en même temps que la conscience tourmentée de ce dernier; c'est parce qu'il sait quelle restera là et qu'elle ne l'abandonnera pas qu'il va se livrer à la police, et c'est sur ses conseils qu'il embrasse la terre contre laquelle il a péché. Sonia, qui amène Raskolnikov à accepter la souffrance, l'accompagne également dans cette souffrance : «Raskolnikov sentit et comprit à cet instant, une fois pour toutes, que Sonia était avec lui à jamais et le suivrait, s'il le fallait, au bout du monde, partout où l'enverrait sa destinée» (Dostoïevski, 1984, p. 600). Sonia veillera toujours sur lui : elle ne lui dirait pas de se dénoncer s'il y avait la peine de mort, comme on vient de l'évoquer, mais c'est le bagne qui attend Raskolnikov, c'est la souffrance rédemptrice pour la terre qu'il a embrassée.

Si Sonia fait naître en Raskolnikov des sentiments de souffrance pour autrui à plusieurs reprises et le transforme finalement, elle agit également sur l'autre criminel du roman, Svidrigailov, à qui, là encore, elle ne veut que du bien (qu'on pense à la scène où Svidrigaïlov vient voir Sonia avant de se tuer, et où, comme il la quitte sans qu'elle ait deviné ses projets, Sonia s'inquiète pour lui car il sort sous la pluie) : en effet, c'est après la rencontre avec Sonia que Svidrigaïlov est capable d'une bonne action, lorsqu'il lui donne de l'argent.

Sonia apporte l'idée d'un salut terrestre aux criminels, un salut qui passe par l'acceptation de la 
souffrance et par le rachat de ses péchés sur la terre pour les hommes. Mais qu'en est-il du salut céleste? serons-nous tous sauvés, les meilleurs comme les pires?

On trouve une réponse à cette question dans la bouche du père de Sonia dès le début du roman, lors de la rencontre de Marmeladov et Raskolnikov. Marmeladov parle en effet de Sonia et dit à Raskolnikov la certitude qu'il a que sa fille sera sauvée; puis il ajoute, à propos de Dieu (Dostoïevski, 1984, p. 51) :

Il jugera tous les hommes et les pardonnera, les bons et les mauvais, les sages et les humbles... Et quand il aura fini avec eux tous, alors il s'adressera aussi à nous : 'Avancez, vous aussi! Avancez, les poivrots, avancez les faiblards, avancez, les impudiques!' Et nous avancerons tous, sans honte, et nous dresserons devant lui. Et il dira: 'Vous êtes des cochons. [...] Mais venez, vous aussi!'

Un pardon pour tous les hommes quels qu'ils soient : tel est le souhait de Dostoïevski qui transparaît à travers le roman. C'est ce souhait dont Sonia se fait l'écho, elle qui invite les criminels à se racheter par la souffrance, mais par une souffrance qui leur permettra de se racheter à la fois aux yeux des hommes et aux yeux de Dieu, même si nous sommes déjà tous sauvés pour l'autre vie. C'est cette même idée qui resplendira encore dans Les Frères Karamazov à travers la voix du paysan qui conduit Dmitri Karamazov; en effet, lorsque ce dernier lui demande s'il ira en enfer, le paysan lui répond, en parlant de Jésus, qu'il

a délivré tous les damnés qui y souffraient (en enfer). Et l'enfer a gémi, croyant que personne ne viendrait plus, c'est-à-dire les damnés. Et le Seigneur a dit alors à l'enfer : «Ne gémis pas, enfer, car d'ici viendront chez toi toutes sortes de grands seigneurs, d'administrateurs, de grands juges et de riches, et tu seras tout aussi plein que tu létais aux siècles des siècles, jusquà ce que je vienne de nouveau». C'est sûr, il y a eu une parole comme ça... (Dostoïevski, 2009, pp. 472-473)

Même le diable, lit-on dans Les Frères Karamazov lors de la vision d'Ivan Karamazov, sera racheté et deviendra bon (ce que l'on retrouve également chez
Victor Hugo). On ne saurait aller plus loin dans la foi en un Dieu de pardon et d'amour.

Pourquoi alors la souffrance terrestre du criminel? Quel est le sens de la vie terrestre si nous sommes déjà tous sauvés? Et pourquoi les hommes seraient-ils bons s'ils peuvent faire ce que bon leur semble, s'ils ont la certitude du salut? C'est que, justement, le salut universel et l'amour de Dieu pour les hommes doivent faire prendre conscience à ces derniers de la nécessité d’être bons, et que quiconque a compris l'amour que Dieu porte aux hommes ne peut pas ne pas être bon. L'homme bon ne veut pas que le mauvais soit puni (il deviendrait mauvais par ce seul désir), il veut au contraire que le mauvais devienne bon. Pour Raskolnikov, devenir bon passe par la souffrance du bagne, en plus de la souffrance psychologique après les meurtres qu'il a commis; pour Svidrigaillov, c'est une prise de conscience du mal qu'il a fait qui le tourmente et qui le pousse à se tuer, mais c'est là la mauvaise voie : Dieu ne veut jamais la mort du pécheur, ni sur terre ni au Ciel. Svidrigaïlov est également sauvé pour l'autre vie, mais il aurait dû vivre sa vie terrestre jusqu'au bout et se racheter dans la souffrance acceptée. Raskolnikov accepte cette souffrance, Svidrigailov la refuse; mais il n'y a en fin de compte que des hommes sauvés. Dostoïevski ne conçoit pas de salut qui ne soit pas universel : la joie des bons est de voir les mauvais sauvés également, leur justice consiste à les aider à devenir bons et à accepter de vivre sur terre en se rachetant. Car la vie humaine est sacrée, c'est ce que rappelle sans cesse Sonia, et le sens à lui donner est d'être bon et d'aider les mauvais à devenir bons également.

Crime et châtiment répond ainsi à un ensemble de questions qui tourmentent Dostoïevski. La femme idéale, Sonia, est bonne jusqu'à l'extrême, et donne sa vie pour les autres en permanence; tous les hommes seront sauvés, et la souffrance terrestre est rédemptrice pour vivre sur terre, ce dont Raskolnikov a conscience et ce qui le fait se livrer à la police; mais c'est la souffrance et non la mort par laquelle doit passer le criminel : la mort est toujours repoussée, qu'il s'agisse de la mort terrestre (jamais personne ne doit mourir, et on sait que Dostoïevki 
était tout à fait opposé à la peine de mort) ou de la mort céleste. On peut supposer que l'homme méchant qui n'aura pas racheté le mal qu'il a fait sur terre le rachètera dans l'au-delà, mais dans le purgatoire, car ce sera une souffrance purifiante qui lui permettra de rejoindre le Ciel par la suite : il n'y a pas d'enfer chez Dostoïevski, simplement un purgatoire. Quant à la vie de l'homme bon, elle ne consiste pas à vouloir du mal à l'homme mauvais, mais à lui vouloir du bien et à le ramener à la vie bonne, comme le montre la joie de Sonia lorsque, au bagne, Raskolnikov se jette en pleurs à ses pieds: «Un bonheur infini brilla dans ses yeux; elle avait compris, il ne pouvait plus y avoir de doute pour elle : il l'aimait, il l'aimait sans bornes, enfin était arrivée cette minute...», et «(l)'amour les avait ressuscités» (Dostoïevski, 1984, p. 625). L'amour et la souffrance sont rédempteurs sur terre, et la prise de conscience d'avoir fait le mal fait infiniment souffrir : c'est de repenser au mal qu'il a fait à Sonia qui a tourmenté Raskolnikov, mais qui ne le tourmente plus à la fin puisqu'il a décidé de le racheter par l'amour : «il savait de quel amour infini il rachèterait maintenant tout ce qu'il lui avait fait souffrir», et c'est cet amour qui le pousse à se demander finalement s'il ne veut pas croire en l'Evangile comme celle qu'il aime : «Estil possible maintenant que ses convictions ne soient pas aussi les miennes? Ses sentiments du moins, ses aspirations...» (Dostoïevski, 1984, p. 626). Michel Eltchaninoff (2011, p. 87) résume bien l'idéal de justice qui est déjà sous-jacent dans Crime et châtiment, avant même le «nous sommes tous coupables pour tous et pour tout » des Frères Karamazov:

Enfin, un idéal normatif émerge qui propose à la justice un principe d'humanité paradoxal, la culpabilité de chacun devant tous et pour tout. Il vise à remplacer l'ethos de la vengeance ou celui de lobjectivité scientifique du jugement par une éthique qui relie la réalité du mal, la nécessité du châtiment, la possibilité de s’amender mais également de pardonner.

Certes, dans Crime et châtiment, nous ne sommes pas encore dans la culpabilité universelle pour tous et pour tout, mais déjà est présente la nécessité du rachat sur terre, le rejet de la peine de mort et l'inconditionnalité du salut divin. Quant à la question du mal, la réponse qu'elle reçoit est parfaitement décrite par Daniel Lefevre d'après nous, qui comprend tout à fait dans les lignes qui suivent le sentiment de Dostoïevski (Lefevre, 2007, p. 28): « le mal n'est pas seulement une question philosophique à résoudre, il est une donnée fondamentale de la condition humaine à laquelle on ne peut répondre que par une attitude d'universelle pitié s'adressant aussi bien à celui qui est cause de la souffrance quà celui qui en est la victime. »

C'est tout le sens de la vie, de laquelle Raskolnikov (le «schismatique» en russe, comme on le rappelle souvent) s'était éloigné, qui lui est révélé par Sonia, et c'est, en même temps, le sens de la souffrance et la certitude de la possibilité de la bonté sur terre et du salut universel qui s'affirme dans Crime et châtiment, roman «d'un homme qui s'efforçait d'exprimer sous la forme la plus convaincante les sentiments et les idées dont il vivait» (Pascal, 1984, p. 24).

\section{Bibliographie :}

Catteau, J. (2000). Dostö̈evski. Correspondance, tome 2 (édition intégrale, présentée et annotée par Jacques Catteau, traduction par Anne Coldefy-Faucard). Paris: Éditions Bartillat.

De Grève, C. (1998). "Crime et châtiment. Roman d'un crime ou roman du crime ", Op. cit. Revue de littératures française et comparée, 11, pp. 305-313.

Dostoïevski, F. (1999). Les Carnets de la maison morte (traduction d'André Markowicz). Paris : Actes Sud.

Dostoïevski, F. (1984). Crime et châtiment (traduction et préface de Pierre Pascal). Paris : GF Flammarion.

Dostoïevski, F. (2009). Les Frères Karamazov (traduction d'Elisabeth Guertik). Paris : Le Livre de poche.

Dostoïevski, F. (1972). L'Idiot, tomes 1 et 2 (traduction de G. et G. Arout). Paris : Librairie générale française.

Dostoïevski, F. (2000). Netotchka Nezvanova (traduction d'André Markowicz). Paris : Actes Sud. 
Dostoïevski, F. (2010). Un petit héros, in Le songe d'un homme ridicule et autres récits (préface de Michel Aucouturier et traduction de Gustave Aucouturier). Paris : Gallimard.

Eltchaninoff, M. (2011). « «Coupable devant tous et pour tout». Justice et culpabilité chez Dostoïevski », Etudes, 414, pp. 77-87.

Jouve, V. (2001). Poétique des valeurs. Paris : PUF.

Lefevre, D. (2007). "Sur les pas de Raskolnikov », Imaginaire \& inconscient, 19, pp. 25-29.

Leichter-Flack, F. (2008). " Encore un mal que le roman nous fait? Morale du dilemme, de Hugo à Dostoievski », Romantisme, 142, pp. 71-81.

Martínez Fernández, I. (2003). «Unas palabr as sobre Pobres gentes », Eslavística complutense, 3, pp. 57-65. 